\title{
General Method for Classicality Certification in the Prepare and Measure Scenario
}

\author{
Carlos de Gois $\odot,{ }^{1}$ George Moreno $\odot,{ }^{2}$ Ranieri Nery, ${ }^{2}$ Samuraí Brito $\odot,{ }^{2}$ Rafael Chaves, ${ }^{2,3}$ and \\ Rafael Rabelo ${ }^{1, *}$ \\ ${ }^{1}$ Instituto de Física "Gleb Wataghin", Universidade Estadual de Campinas, Campinas CEP 13083-859, Brazil \\ ${ }^{2}$ International Institute of Physics, Federal University of Rio Grande do Norte, Natal 59070-405, Brazil \\ ${ }^{3}$ School of Science and Technology, Federal University of Rio Grande do Norte, Natal 59078-970, Brazil
}

(Received 13 January 2021; revised 28 April 2021; accepted 22 June 2021; published 20 July 2021)

\begin{abstract}
Preparing and measuring physical systems are the operational building blocks of any physical experiment, and to describe them is the first purpose of any physical theory. Remarkably, even when only uncharacterized preparation and measurement devices are present, it is sometimes possible to distinguish between the behaviors of quantum and classical systems from only observational data. Certifying the physical origin of measurement statistics in the prepare and measure scenario is of primal importance for developing quantum networks, distributing quantum keys, and certifying randomness, to mention a few applications, but, surprisingly, no general methods to do so are known. We progress on this problem by crafting a general, sufficient condition to certify that a given set of preparations can only generate classical statistics, for any number of generalized measurements. As an application, we employ the method to demonstrate nonclassicality activation in the prepare and measure scenario, also considering its application in random access codes. Following that, we adapt our method to certify, again through a sufficient condition, whether a given set of measurements can never give rise to nonclassical behaviors, irrespective of what preparations they may act upon. This, in turn, allows us to find a large set of incompatible measurements that cannot be used to demonstrate nonclassicality, thus showing incompatibility is not sufficient for nonclassicality in the prepare and measure scenario.
\end{abstract}

DOI: 10.1103/PRXQuantum.2.030311

\section{INTRODUCTION}

Quantum theory, albeit ubiquitous and tested extensively, still presents us with interesting and unintuitive phenomena even for the simplest physical systems. The paradigmatic examples are Bell nonlocality $[1,2]$ and Einstein-Podolsky-Rosen (EPR) steering [3-5] - manifest as strong correlations between spacelike separated experiments performed by independent observers - that can unambiguously discern between classical and quantum predictions. More recently, a similar division was found to arise in a setup closely related to quantum communication tasks, the so-called prepare and measure (PAM) scenario [6].

Descriptions of the preparation of physical systems and of their measurements are the building blocks of

*rabelo@ifi.unicamp.br

Published by the American Physical Society under the terms of the Creative Commons Attribution 4.0 International license. Further distribution of this work must maintain attribution to the author(s) and the published article's title, journal citation, and DOI. any physical theory. Hence, it is notable that even in a semi-device-independent approach-relying alone on observational data and mild assumptions about the state preparation - such a scenario is already enough to distinguish between quantum and classical behaviors. Apart from its foundational relevance, certifying that the systems employed are indeed quantum and behave as expected is an essential task in applications of the PAM scenario, ranging from communication in quantum networks $[7,8]$ and self-testing $[9,10]$ to quantum key distribution [11], randomness certification [12], and random access codes (RACs) [13], while also figuring at the core of informational principles for quantum theory $[14,15]$ and the modeling of paradigmatic gedanken experiments such as the so-called delayed choice experiment [16].

It has been long known that Holevo's bound [17] limits the amount of information that may be retrieved in such a scenario, implying that quantum messages cannot transmit more information than their classical counterparts. Notwithstanding, the nonclassicality of the measurement outcomes can still be witnessed if one imposes certain assumptions on the preparation device [6,18-22]. For instance, even though a qubit can transmit at most a bit 
of information, it can still generate measurement statistics that cannot be reproduced by a classical bit $[6,18]$. On the more applied side, such dimension assumptions can also be used to derive device-independent witnesses for the Hilbert space dimension of the prepared states [23-25].

A task of primal importance is to be able to decide whether a given set of prepared states can give rise to nonclassical behaviors. In a Bell scenario, for instance, it has been long known that entanglement is a necessary but insufficient ingredient for nonlocality, as there are entangled states that cannot violate any Bell inequality [26,27], giving rise to general methods for deciding whether an entangled state is local $[28,29]$. More than its fundamental relevance, such criteria can also be employed to show hidden nonlocality or activation of nonlocality, the former related to the fact that local states can have their nonlocality activated if one performs local filtering prior to measurements [30-32] and the latter related to measurements on a number of copies of such states [33-35]. In PAM scenarios, however, in spite of their relevance, the question of whether a given set of preparations can lead to nonclassical behaviors is yet to be investigated in more depth. At this point, it is worth emphasizing that, in this paper, we strictly refer to the notion of PAM classicality as that defined in [6] and several of the previously cited references, a precise definition of which is given in the following section. It is also worth mentioning that similar notions are studied in the formalism of ontological models [36]. Within this formalism, several results have been obtained lately regarding the observation of nonclassicality in quantum theory and, more generally, in generalized probabilistic theories [37-42]. In a more applied side, such nonclassicality has been identified as a resource in state discrimination tasks [43]. Despite similarities, the definitions of nonclassicality adopted within this formalism differ considerably from that adopted in this paper: whereas in the former one usually associates classicality with noncontextuality, in the latter, classicality is associated with the existence of a different kind of ontological model.

Regarding the PAM scenario, it was only recently that the first test, though of limited applicability, has been proposed in order to detect nonclassicality - in the strict sense discussed previously and yet to be precisely defined - of quantum state preparations [22]. Strikingly, however, as opposed to the Bell case, no general methods are known to provide such certification. That is precisely the problem we solve in this paper. Inspired by results crafted for constructing local hidden variable models for entangled states $[28,29]$, we propose a general method allowing us to certify whether the behaviors arising from a preparation set are always classically reproducible, irrespective of what generalized measurements are applied on them. Taking the measurements as the resource, we conversely derive a method to test whether a measurement set can be classically reproduced no matter what preparations they act upon. We demonstrate the applicability of both methods in a number of cases, and in particular employ them to show activation of nonclassicality of a given set of states, and also prove the insufficiency of measurement incompatibility for observing nonclassical behaviors in the PAM scenario.

\section{PREPARE AND MEASURE SCENARIO}

In the PAM scenario, a preparation device $P$ takes a random variable $x \in \mathcal{X}$ as input and, subjected to it, prepares a physical system in a specific physical state. A second device, $M$, receives the prepared system and, given an independently chosen random variable $y \in \mathcal{Y}$ as the choice of an observable, measures the system returning the output $b \in \mathcal{B}$ [see Fig. 1(a)]. Without further information on the inner workings of these devices, the best possible description is given by the probabilities $p(b, x, y)$ of the observed events. Without loss of generality, and given that the state preparation $x$ and measurement choices $y$ are under control of the experimenters, one typically considers the conditional distribution $p(b \mid x, y)$ represented by the set of behaviors $\mathbf{p}=\{p(b \mid x, y)\}_{b, x, y}$

Classically, the state being prepared by the device $P$ is a random variable $a \in \mathcal{A}$, that can be understood as a message being sent to the $M$ device. Assuming that the preparation and measurement devices might have some preshared correlations, described by the random variable $\Lambda$ governed by a probability distribution $\pi$ (lambda), the most general classical description of this experiment is given by

$$
p(b \mid x, y)=\sum_{a} \int_{\Lambda} \pi(\lambda) p(a \mid x, \lambda) p(b \mid a, y, \lambda) .
$$

To obtain the decomposition above, a set of causal assumptions is considered. First the independence of the preshared correlations from the state preparation and measurement choices, that is, $p(x, y, \lambda)=p(x, y) p(\lambda)$; an assumption (a)

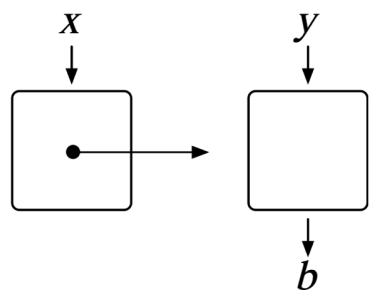

(b)

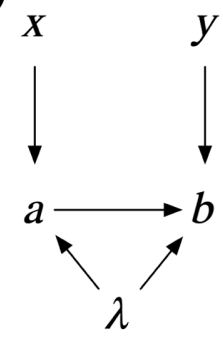

FIG. 1. Operational and causal representations of a PAM scenario. On the left, the black-box shows a preparation, conditioned on input $x$, being sent to a measurement device with measurement choice $y$ and output $b$. On the right, the DAG turns explicit the unobservable variables $a$ and $\lambda$ in the causal structure. 
that in the context of Bell scenarios is referred as measurement independence or "free-will" [44-46]. The second causal assumption follows from the fact that, differently from a Bell scenario, in the PAM case we deal with temporal correlations. More precisely, the measurement device lies in the future light cone of the preparation device. Thus, the message $a$ should only be causally dependent on the input $x$ and the shared correlations $\lambda$. The final causal assumption is given by the fact that even though the outcome $b$ can depend on the input $x$, such correlations are mediated by the state being prepared, that is, the correlations between $b$ and $x$ are screened-off once we condition on the values of $a$ and $\lambda$. Such causal assumptions can be faithfully and graphically represented using the intuitive directed acyclic graph (DAG) shown in Fig. 1(b). In a quantum description, states from the set $\mathcal{S}=$ $\left\{\rho_{x}\right\}_{x}$ are prepared and the possible observables are quantum measurements $\mathcal{M}_{y}$, where each $\mathcal{M}_{y}=\left\{M_{b \mid y}\right\}_{b}$ is a collection of positive operator-valued measure (POVM) operators. The quantum experiment is then defined by $\mathcal{E}=$ $\{\mathcal{S}, \mathcal{M}\}$, where $\mathcal{M}=\left\{\mathcal{M}_{y}\right\}_{y}$. Employing Born's rule any element $p(b \mid x, y)$ in the behavior $\boldsymbol{p}$ of experiment $\mathcal{E}$ is then given by

$$
p(b \mid x, y)=\operatorname{tr}\left(M_{b \mid y} \rho_{x}\right) .
$$

Note that if $|\mathcal{A}| \geq|\mathcal{X}|$, a message $a$, even if classical, may perfectly encode the choice $x$ of preparation, and any behavior $\boldsymbol{p}$ is then reproducible with no more than classical communication. Only when some restriction is imposed on the amount of communication, is that differences between the classical and quantum predictions can emerge [6,18-22]. Typically, then, the bound $|\mathcal{A}|<$ $|\mathcal{X}|$ is imposed on the dimension of the classical message. When each and every $p(b \mid x, y)$ can be written as the classical model (1), the quantum experiment $\mathcal{E}$ is classically simulatable, meaning that the measurements statistics can be obtained by transmitting $|\mathcal{A}|$-dimensional classical messages. In principle, nothing precludes $|\mathcal{A}|$ from being different from the dimension of the quantum preparations.

\section{WITNESSING THE CLASSICALITY OF PREPARATIONS}

The first question one might ask in the PAM scenario is whether a quantum experiment $\mathcal{E}$, characterized by prepared states and measurements, can be classically simulated. Similarly to what happens in a Bell scenario, if the cardinalities of the sets of the variables $a, b, x$, and $y$ are fixed, the set of behaviors compatible with the classical description (1) is a polytope that can be characterized in terms of a finite number of linear (Bell-like) inequalities. If one has a complete description of such inequalities for a given scenario, then the compatibility of the quantum experiment data with a classical description can be easily certified if no inequalities are violated. The problem of this approach is twofold. First, determining all inequalities becomes intractable as we increase the cardinality of the variables. The second, more fundamental issue, stems from the fact that states that can only give rise to classical correlations if a given number of measurements are performed, can indeed have their nonclassicality revealed if we increase the number of possible measurements [22]. In the following Theorem, we address this problem by deriving a sufficient condition to certify the classicality of a given set of prepared states valid for any number of projective measurements (PMs).

Theorem 1 (Preparation classicality for all PMs). Let $\mathcal{S}=\left\{\rho_{x}\right\}_{x=1}^{|\mathcal{X}|}$ be a set of d-dimensional quantum preparations and $\mathcal{M}$ be a finite set of projective, rank-1 measurements on $\mathbb{C}^{d}$. Each projector in $\mathcal{M}$ is associated to a generalized Bloch vector, in a generalized Bloch sphere. Let $\eta$ be the radius of the largest sphere inscribed in the convex hull of these vectors associated to the projectors in $\mathcal{M}$. Define, for each $x \in\{1, \ldots,|\mathcal{X}|\}$, the operators

$$
O_{x}=\frac{1}{\eta}\left[\rho_{x}-(1-\eta) \frac{\mathbf{1}_{d}}{d}\right] .
$$

If, for all $O_{x}$ and $M_{b \mid y} \in \mathcal{M}$, the behavior $\mathbf{p}=$ $\left\{\operatorname{tr}\left(M_{b \mid y} O_{x}\right)\right\}_{b, x, y}$ can be reproduced by communicating $|\mathcal{A}|$-dimensional classical systems [Eq. (1)], then the set $\mathcal{S}$ of preparations is classically simulatable for all PMs.

Proof. We begin by recalling that each $\Pi_{b \mid y} \in \mathcal{M}$ may be associated to a unit vector $\boldsymbol{v}_{b \mid y} \in \mathbb{R}^{\left(d^{2}-1\right)}$ by means of

$$
\Pi_{b \mid y}=\frac{1}{d}\left(\mathbf{1}_{d}+c_{d} \sum_{i=1}^{d^{2}-1}\left(v_{b \mid y}\right)_{i} \sigma_{i}\right),
$$

where $c_{d}=\sqrt{d(d-1) / 2}$ and $\left\{\sigma_{i}\right\}_{i}^{\left(d^{2}-1\right)}$ is a set of $d \times d$ traceless operators generators of the $S U(d)$ groupthat together with $\mathbf{1}_{d}$, form an orthonormal basis for the space of $d \times d$ linear operators (with respect to the HilbertSchmidt inner product).

With that in mind, let $\mathcal{M}=\left\{\Pi_{b \mid y}\right\}_{b, y}$ be a finite set of PMs, where each $\Pi_{b \mid y}$ is a rank-1 projector (we later show that this leads to no loss of generality), and $\sum_{b} \Pi_{b \mid y}=$ $\mathbf{1}_{d}, \forall y$. We write $\eta$ for the radius of the largest sphere that can be inscribed into $\operatorname{conv}(\mathcal{M})=\operatorname{conv}\left(\left\{\boldsymbol{v}_{b \mid y}\right\}_{b, y}\right)$ and $\left\{O_{x}\right\}_{x=1}^{|\mathcal{X}|}$ for the set of operators implicitly defined by $\rho_{x}=$ $\eta O_{x}+(1-\eta)\left(\mathbf{1}_{d} / d\right)$.

When model (1) exist for preparations $\mathcal{S}$ and measurements $\mathcal{M}$, it also does for any convex combination of the operators in $\mathcal{M}$. In particular, then, classicality follows for any measurements in the largest sphere that can be inscribed into $\operatorname{conv}(\mathcal{M})$ (see Fig. 2 for a representation in $d=2$ ). As any measurement operator on such a 


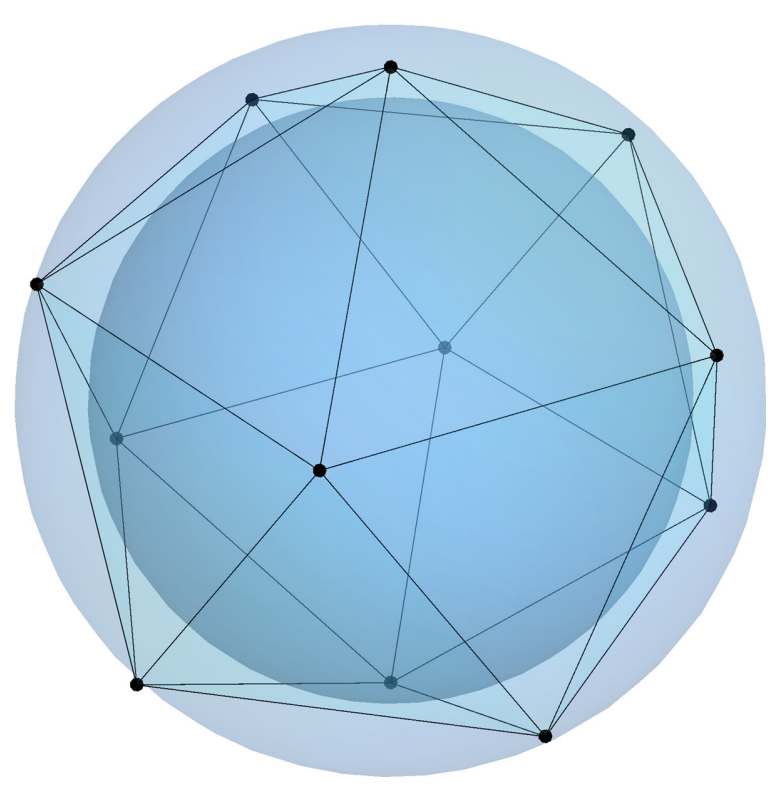

FIG. 2. Representation of the method for $d=2$. Each vertex on the Bloch sphere represents a measurement operator. To every $\Pi_{0 \mid y}$ we associate a corresponding antipodal $\Pi_{1 \mid y}$. Measurements inside the set enclosed by $\operatorname{conv}(\mathcal{M})$ can be simulated by mixing these extremal ones. In particular, any measurement $\left\{\Pi_{b \mid u}^{\eta}\right\}_{b}$ in a ball with radius $\eta$ inscribed in the polytope is simulatable in such manner.

sphere is related to a valid rank-1 projector $\Pi_{b \mid u}$ through $\Pi_{b \mid u}^{\eta}=\eta \Pi_{b \mid u}+(1-\eta) \mathbf{1}_{d} / d$, a simple calculation yields

$$
\operatorname{tr}\left(O_{x} \Pi_{b \mid u}^{\eta}\right)=\operatorname{tr}\left(\rho_{x} \Pi_{b \mid u}\right), \quad \forall x, b, u
$$

Equation (4) tells us the result of applying any projection $\Pi_{b \mid u}$ to a density operator $\rho_{x}$ is equivalent to applying a depolarized projection $\Pi_{b \mid u}^{\eta}$ to $O_{x}$ - an inflated instance of $\rho_{x}$.

With that in mind, suppose we probe each $O_{x}$ with every $\Pi_{b \mid y} \in \mathcal{M}$ and find the observed behavior has a classical model as given by Eq. (1). In that case, as already noted, the model would exist for all $\Pi_{b \mid u}^{\eta}$, as they all can be written as convex combinations of the $\Pi_{b \mid y}$. Thereon, Eq. (4) affirms the $\rho_{x}$ are classically simulatable for all rank-1 projectors.

To see this condition is also valid for arbitrary projections, first note that in $\mathbb{C}^{2}$ all nontrivial projections are unit-rank. Even though this is not true for $d>2$, it is true that any PM can be seen as a rank-1 PM with coarse graining. Hence, the result holds for all PMs.

Strikingly, by probing the statistics of only finitely many measurements, Theorem 1 provides us with a sufficient condition to certify that the set of prepared states $\mathcal{S}=\left\{\rho_{x}\right\}_{x=1}^{|\mathcal{X}|}$ can only exhibit classical correlations, even if infinitely many PMs were to be performed. This sufficient condition for classicality also becomes necessary when $\eta=1$, in which case we recover the brute-force approach of testing all possible measurements. In addition, this classicality condition can be expressed as the following feasibility problem

$$
\begin{aligned}
\text { given } & \mathcal{S}, \mathcal{M}, \eta,\{\lambda\} \\
\text { find } & \pi(\lambda) \\
\text { s.t. } & \rho_{x}=\eta O_{x}+(1-\eta) \frac{\mathbf{1}_{d}}{d}, \forall x \\
& \operatorname{tr}\left(\Pi_{b \mid y} O_{x}\right)=\sum_{a, \lambda} \pi(\lambda) p(a \mid x, \lambda) p(b \mid a, y, \lambda) \\
& \quad \forall b, x, y \\
& \pi(\lambda) \geq 0, \quad \\
& \sum_{\lambda} \pi(\lambda)=1
\end{aligned}
$$

which is a linear program and thus can be solved efficiently. In particular, condition (5d) enforces the existence of the classical model (1), and Fine's theorem was used to cast the integral as a finite sum [47].

Dichotomic PMs are the extremal two-effect POVMs, so Theorem 1 is also a condition for classicality under all such measurements. To extend our result to generalized measurements, we observe that any POVM collection $\mathcal{M} \subseteq$ $\mathcal{P}(d, n)$ where $\mathcal{P}(d, n)$ is the set of generalized measurements with $n$ effects acting on $d$-dimensional preparations, can be simulated by PMs and classical processing after a certain amount $t$ of depolarization on its effects [48], which leads to the following extension of Theorem 1.

Theorem 2 (Preparation classicality for all POVMs). Let $\Phi_{t}(\mathcal{M})$, where $\Phi_{t}(\cdot)=t(\cdot)+(1-t)(\operatorname{tr}(\cdot) / d) \mathbf{1}_{d}$ is a depolarizing channel acting on the effects of $\mathcal{M}$, be projective-simulatable for any $\mathcal{M} \subseteq \mathcal{P}(d, n)$. If preparation $\rho^{\prime}=(1 / t)\left\{\rho-[(1-t) / d] \mathbf{1}_{d}\right\}$ has a classical model for all PMs, then $\rho$ is classically reproducible for all POVMs.

Proof. Let $\rho^{\prime}$ be such that probabilities $\operatorname{tr}\left(\Pi_{b \mid y} \rho^{\prime}\right)$ admit description (1) for all projections $\Pi_{b \mid y}$, and $\mathcal{M}=\left\{M_{b \mid y}\right\}_{b, y}$ be a collection of POVMs. As the statistics generated by $\Phi_{t}(\mathcal{M})$ for any $\mathcal{M} \subseteq \mathcal{P}(d, n)$ can be reproduced by PMs and classical processing, it must be that the behavior $\left\{\operatorname{tr}\left[\Phi_{t}\left(M_{b \mid y}\right) \rho^{\prime}\right]\right\}_{b, y}$ is also classically reproducible. But $\Phi_{t}(\cdot)$ is self-dual, hence $\operatorname{tr}\left[\Phi_{t}\left(M_{b \mid y}\right) \rho^{\prime}\right]=$ $\operatorname{tr}\left[M_{b \mid y} \Phi_{t}\left(\rho^{\prime}\right)\right]=\operatorname{tr}\left(M_{b \mid y} \rho\right), \forall b, y$. Therefore, $\rho$ has a classical model for all POVMs.

Accordingly, showing that preparations $\rho_{x}$ have a classical description for all POVMs is equivalent to proving that preparations $\rho_{x}^{\prime}=(1 / t)\left\{\rho_{x}-[(1-t) / d] \mathbf{1}_{d}\right\}$ are classically reproducible for all PMs, which can be done 
by providing $t$ and the relevant additional restrictions to program (5). For $d=2$, the POVM $A_{\text {tetra }}=\left\{\frac{1}{4}\left(\mathbf{1}+\boldsymbol{v}_{i} \cdot \sigma\right)\right\}_{i}$ where $\boldsymbol{v}_{i}$ are the vertices of a regular tetrahedron, have the highest noise robustness, thus any $t$ such that $\Phi_{t}\left(A_{\text {tetra }}\right)$ is projective-simulatable also makes so any $A \in \mathcal{P}(2, n)$. For qubits, $t=\sqrt{2 / 3}-\epsilon$ suffices [49]. On higher $d$, $t=1 / d$ is a lower bound on $t$ that can be tightened through semidefinite programming [48].

\section{A. Computational analysis}

The computational bottleneck of program (5) lies in the fact that decomposing the deterministic strategies $\lambda$ into their extremal points results in an exponentially large set, with $N_{\lambda} \propto|\mathcal{B}|^{|\mathcal{A}||\mathcal{Y}|}$ extremal points. Consequently, regardless of the efficiency of linear programming algorithms, the size of program (5) scales exponentially in the number of measurements. In principle, this precludes us from using too large sets of measurements, and consequently obtaining larger values of $\eta$, where the drawback of a small $\eta$ is that we will only be able to certify classicality for reasonably mixed states. However, this issue can be circumvented by adapting the procedure outlined in [50].

Our strategy will be to avoid working on all $N_{\lambda}$ extremal points at once, and instead iteratively exploring the deterministic strategy space. Notice that the factibility program (5) may be equivalently written as a maximization program,

$$
\begin{aligned}
\text { given } & \mathcal{S}, \mathcal{M}, \eta,\{\lambda\} \\
\max _{\pi(\lambda)} & \alpha \\
\text { s.t. } & \alpha \rho_{x}+(1-\alpha) \frac{\mathbf{1}_{d}}{d}=\eta O_{x}+(1-\eta) \frac{\mathbf{1}_{d}}{d}, \forall x \\
& \operatorname{tr}\left(\Pi_{b \mid y} O_{x}\right)=\sum_{a, \lambda} \pi(\lambda) p(a \mid x, \lambda) p(b \mid a, y, \lambda), \\
& \forall b, x, y \\
& 0 \leq \alpha \leq 1 \\
& \pi(\lambda) \geq 0 \\
& \sum_{\lambda} \pi(\lambda)=1 .
\end{aligned}
$$

Allowing $\alpha=0$ guarantees a solution will always exist, and obtaining $\alpha=1$ amounts to (5) being factible. Any $\alpha$ in between means that the state $\alpha \rho_{x}+(1-\alpha) \boldsymbol{d}_{d} / d$ is classically simulatable.

One may interpret the program above as a search for the optimal weights $\pi(\lambda)$ such that a convex combination of extremal points of the local polytope describe the behavior of our system. Carathéodory's theorem [51] states that at most $d+1$ extremal points are necessary to optimally describe any point of a $d$-dimensional convex set, hence most of the $\pi(\lambda)$ found will be zero. We cannot know, beforehand, which points make for an optimal description, so we take $N_{\lambda}^{\prime} \gg d+1$ - but much smaller than $N_{\lambda}$ - points and optimize over them. To set up the next iteration, all $\pi(\lambda)=0$ in this result can be discarded and replaced by previously unexplored deterministic strategies, and we run program (6) again. As, at each round, we are keeping all optimal $\lambda$ from the previous round, the optimal value $\alpha$ will be nondecreasing between iterations. Furthermore, for so large $|\mathcal{Y}|$ that it would be prohibitive to enumerate and keep track of all previously visited strategies, we observe that simply randomly sampling $\lambda$ on each run of (6) makes our procedure rapidly converge to $\alpha^{*}$, which assumes a constant value for all subsequent iterations and is interpreted as a lower bound on the maximum visibility imposed on the preparations such that their behavior is classical.

To illustrate the application of Theorem 1 and the procedure just described, we consider the preparation set $\mathcal{S}(\theta, \phi)=\left\{\rho_{\boldsymbol{x}}, \rho_{z}, \rho_{\boldsymbol{r}(\theta, \phi)}\right\}$ that is $|\mathcal{X}|=3$ where $\rho_{\boldsymbol{v}}$ denotes a qubit state with Bloch vector $\boldsymbol{v}$, and the unit vector $\boldsymbol{r}(\theta, \phi)$ is given by its spherical polar and azimuthal angles, respectively. Arranging our measurements operators as those associated to the vertices of an icosahedron $(|\mathcal{Y}|=6$ with $\eta \approx 0.79$ ), we get a fairly small problem that can be solved either directly or iteratively. Figure 3(a) shows the result through the iterative procedure, which exactly matches the direct approach. With twice the amount of measurements arranged as the vertices of a rhombicuboctahedron $(\eta \approx 0.86)$, it is no longer possible to compute 3 (b) in a direct manner. In fact, even enumerating all deterministic strategies becomes too costly, let alone solving the program. Employing the procedure here described allowed us to compute Fig. 3(b). The advantage of having more measurements then becomes evident, with the rhombicuboctahedron resulting in increased visibilities. We pay for that with more computation time: although the model for each preparation set in Fig. 3(a) takes less than a minute to compute on a standard desktop computer, it takes around 7 min for Fig. 3(b) when using fairly robust parameters [likewise for Figs. 5 and 3(b)]. Implementations for these and all forthcoming applications can be found at a public repository [52].

Although our results are fully general, moving on to higher dimensions presents us with two considerable drawbacks. Similarly to the example discussed above, qudits will naturally lead to more extremal points in our classicality polytope, and ultimately to more computation time. Yet another considerable downside is that generating a large depolarized Bloch ball in larger dimensions will be costlier, while also the intuition brought by using polyhedra (with antipodal vertices) as a measurements polytope is lost. Howbeit, picking random 3-qutrits preparations and 12 random PMs (average $\eta \approx 0.26$ ), we found the mean computation time to be around $37 \mathrm{~min}$ for each preparation set. Increasing the number of measurements 
(a)

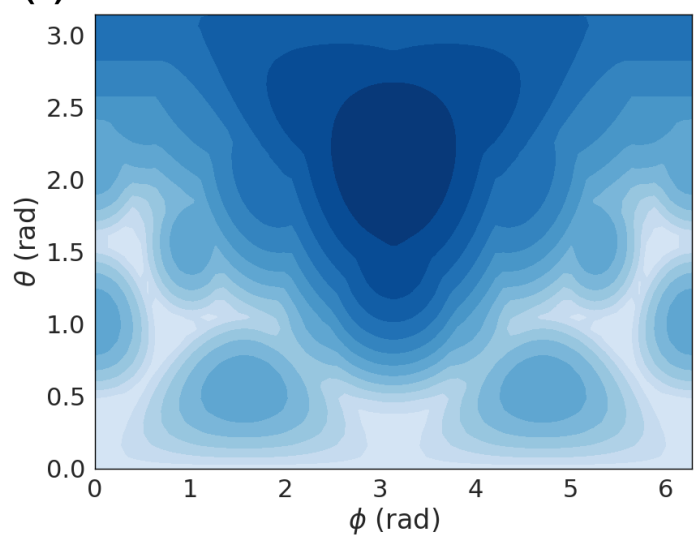

(b)

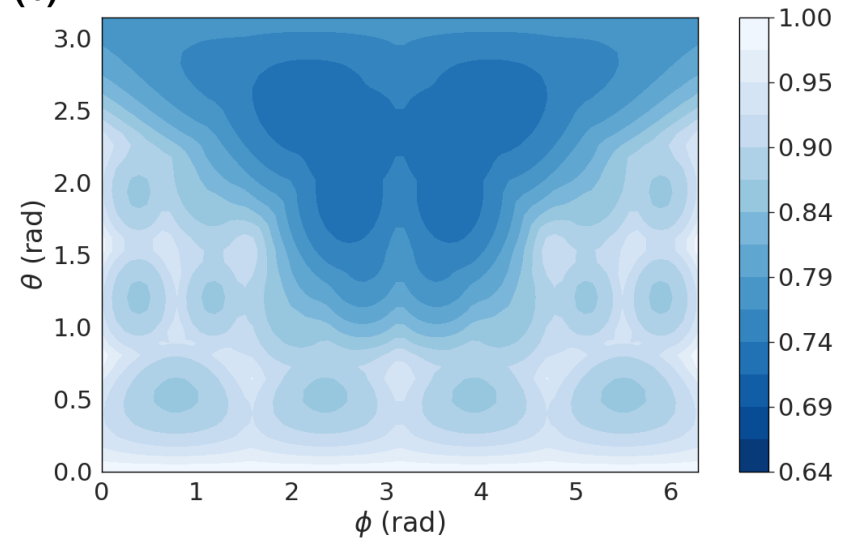

FIG. 3. Application of program (6) to $\mathcal{S}(\theta, \phi)=\left\{\rho_{\boldsymbol{x}}, \rho_{z}, \rho_{\boldsymbol{r}(\theta, \phi)}\right\}$. Levels are the maximum visibility $\alpha$ such that preparation set $\alpha \mathcal{S}(\theta, \phi)$ has a classical model. (a) For the $|\mathcal{Y}|=6$ icosahedron measurements, $\eta \approx 0.79$, and program (6) can be applied directly. (b) A rhombicuboctahedron corresponds to $|\mathcal{Y}|=12 \mathrm{PMs}$ and $\eta \approx 0.86$. As the number of deterministic strategies scales exponentially, the computation is only possible by iteratively optimizing over subsets of deterministic strategies.

and preparations is also possible by paying with more waiting until convergence is reached. In all cases, as each iteration returns a nondecreasing visibility $\alpha$, one will always obtain lower bounds even if convergence is not quickly attained. These observations lead us to argue that, with a clever selection of probe measurements, our method could still be useful for future applications even in larger dimensions.

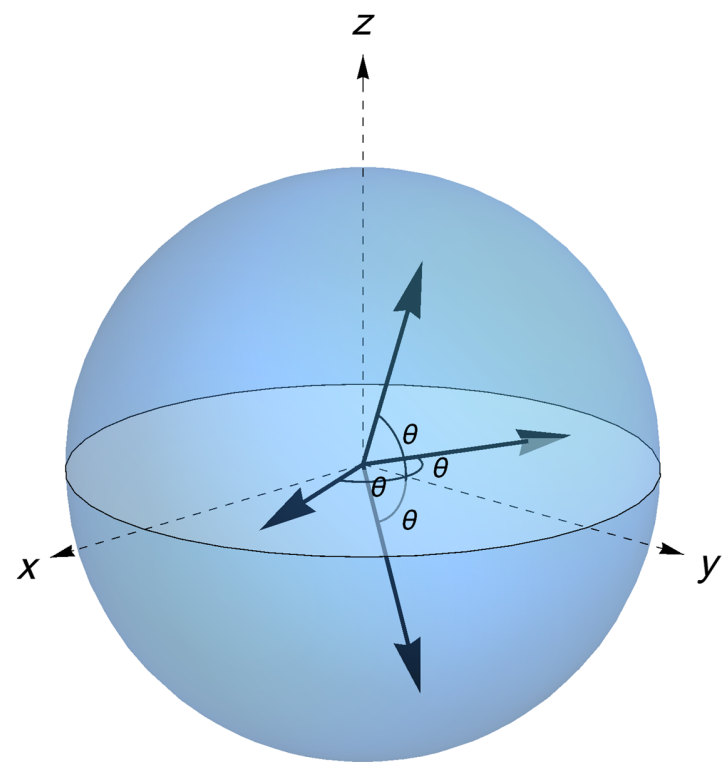

FIG. 4. Preparations $\mathcal{S}(\alpha, \theta)=\left\{\rho_{\boldsymbol{r}_{1}}, \rho_{\boldsymbol{r}_{2}}, \rho_{\boldsymbol{r}_{3}}, \rho_{\boldsymbol{r}_{4}}\right\}$ for $\alpha=0.8$. At $\theta=0$ all preparations are at $\alpha \boldsymbol{y}$. For $\theta=\pi / 2, \mathcal{S}=$ $\{-\alpha \boldsymbol{x}, \alpha \boldsymbol{x},-\alpha \boldsymbol{z}, \alpha \boldsymbol{z}\}$, presenting the largest violation of inequality (7).

\section{B. Nonclassicality activation}

In a Bell scenario, an entangled state that can only lead to local correlations can have its nonlocality activated in at least two manners: with a single copy, by proceeding with local filtering (a phenomenon usually referred as hidden nonlocality) [30-32], or by using many copies of the quantum state [33-35]. In turn, in a PAM scenario a new possibility is open. Consider a set of $n+1$ prepared

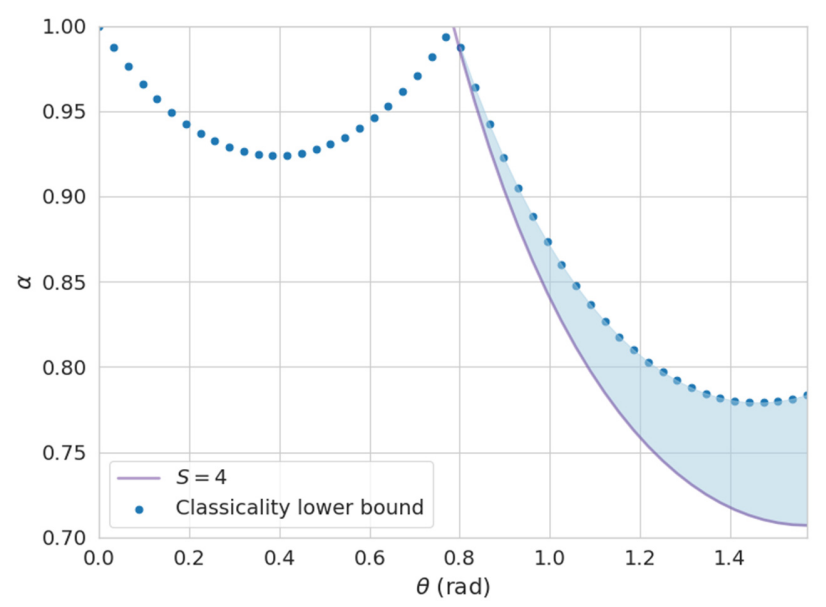

FIG. 5. Nonclassicality activation for the preparations in Fig. 4 and measurements arranged in a rhombicuboctahedron with corresponding $\eta \approx 0.86$. As $S \propto \alpha$, every preparation set above the $S=4$ curve is nonclassical. On the other hand, the blue scatter shows the maximum visibility such that any triad of states in the preparation set are classical. The shaded region thence stands for preparations that exhibit nonclassicality activation by increasing the number of preparations. 
states such that any subset of $n$ of them can only generate correlations describable by the classical model (1). However, if the $n+1$ quantum states can lead to nonclassical correlations then we can say the nonclassicality of the other $n$ states is activated by the preparation of this extra $(n+1)$ th state. For our purposes, it will suffice to consider the case where $n=3$, that is, we have four possible preparations $(|\mathcal{X}|=4)$ such that any combination of only three of them will always lead to classical correlations. All other variables are dichotomic, that is, $|\mathcal{B}|=|\mathcal{A}|=|\mathcal{Y}|=$ 2.

In this specific PAM scenario, there are only two classes of inequalities, the violation of which certify nonclassicality. If we are able to find a set of four states, any three of which only generate classical correlations according to our criteria, but nonetheless violate one of these inequalities when taken together, we would thus have proven the activation of nonclassicality. Towards that end, we consider the inequality given by [11]

$$
\begin{aligned}
S= & E_{11}-E_{12}-E_{21} \\
& +E_{22}-E_{31}-E_{32}+E_{41}+E_{42} \leq 4,
\end{aligned}
$$

where $E_{x y}=p(0 \mid x, y)-p(1 \mid x, y)$ is the expectation value of observable $y$ applied to preparation $x$. When preparations are quantum states and the measurements are extremal, $E_{x y}=\operatorname{tr}\left(M_{0 \mid y} \rho_{x}\right)-\left(M_{1 \mid y} \rho_{x}\right)=\boldsymbol{r}_{x} \cdot \boldsymbol{q}_{y}$, where $\boldsymbol{r}_{x}$, $\boldsymbol{q}_{y}$ are the Bloch vectors that parametrize preparation $x$ and measurement $y$, respectively.

We define $\mathcal{S}(\alpha, \theta)=\left\{\rho_{r_{1}}, \rho_{\boldsymbol{r}_{2}}, \rho_{\boldsymbol{r}_{3}}, \rho_{\boldsymbol{r}_{4}}\right\}$ as the preparation set according to Fig. 4, where $\alpha$ is a shrinking factor from the surface of the Bloch sphere. In turn, we choose PMs parameterized by the vectors $\boldsymbol{q}_{1}=(-\boldsymbol{x}+$ $\boldsymbol{z}) / \sqrt{2}$ and $\boldsymbol{q}_{2}=(\boldsymbol{x}+\boldsymbol{z}) / \sqrt{2}$, for which we obtain that $S=4 \sqrt{2} \alpha \sin \theta$. This shows that, for a large span of $\alpha$ and $\theta$, this family of preparations violate the bound $S \leq 4$, thus exhibiting nonclassicality.

To show this nonclassicality is a genuine nonclassicality activation for dichotomic PMs, we employ our general method to show the behaviors of any subset of three elements of $\mathcal{S}$ are classically reproducible. This was done by applying program (6) with measurements arranged as a rhombicuboctahedron, and corresponding $\eta \approx 0.86$. As a result, for each $\theta$, the optimal value $\alpha^{*}$ stands for the maximum purity of the preparations such that there is a classical model for all triadic subsets of $\mathcal{S}$, and every $\alpha<\alpha^{*}$ represents classical preparations (Fig. 5). As shown in the shaded region, we will have nonclassicality activation for any value $\alpha<\alpha^{*}$ such that inequality $S$ is violated.

\section{Quantum advantage activation in RACs}

These results also have implications for the application of the PAM scenario in RACs. An $(n, d) \mapsto m$ RAC can be understood as a communication task where, at each run, Alice receives a ditstring $\boldsymbol{x}=m_{1} m_{2} \ldots m_{n}$, with each $m_{i} \in\{1, \ldots, d\}$, which she then encodes in $m<n d$-level systems that will be sent to Bob. Upon receiving this limited communication from Alice and an uniformly sampled query $y \in\{1, \ldots, n\}$, Bob is asked to determine the letter $m_{y}$ from Alice's string. Depending on the type of systems used to encode information and the amount of correlations allowed between the parties, several possible implementations of this task arise. Quantum-RACs (QRACs) are those which use quantum systems for communication. They appeared in [53] and were later rediscovered and linked to quantum automata in [54], and several results regarding existence and advantage of QRACs over their classical counterparts rapidly ensued $[11,13,36,55-$ 59].

A PAM scenario can be seen as a physical implementation of a $(n, d) \mapsto 1$ RAC where we associate each preparation in $\mathcal{S}=\{\boldsymbol{x}\}_{\boldsymbol{x}=1}^{d^{n}}$ with the encoding of a unique ditstring $m_{1} m_{2} \ldots m_{n}$. When these preparations are quantum states, we associate the PAM scenario to a QRAC where Bob's guess will be the output of a quantum measurement on the received states. In this way, a $(2,2) \mapsto 1$ QRAC shall be mapped to a PAM scenario with $|\mathcal{X}|=4$ qubit preparations encoding the bitstrings $m_{1} m_{2} \in\{00,01,10,11\}$ and $|\mathcal{Y}|=2$ dichotomic measurements that will be used to guess either $m_{1}$ or $m_{2}$. Likewise, a (classical) RAC of this same sort will be linked to a PAM scenario with $|\mathcal{A}|=2$ possible classical states (i.e., a bit). Usually, the performance of such protocol is measured through the worst-case or the average-case success probabilities. The average success probability of an $(n, d) \mapsto 1$ RAC is written as

$$
p_{\text {suc }}^{\text {avg }}=\frac{1}{n d^{n}} \sum_{m_{1}, \ldots, m_{n}, y} p\left(b=m_{y} \mid m_{1}, \ldots, m_{n}, y\right),
$$

where the factor $1 / n d^{n}$ comes from assuming that the input dits as well as the variable $y$ are uniformly distributed.

As shown in [11], the average probability of success of an $(2,2) \mapsto 1$ RAC can be directly linked with the inequality $S^{\prime}=E_{11}+E_{12}+E_{21}-E_{22}-E_{31}+E_{32}-$ $E_{41}-E_{42} \leq 4$ in a way that $p_{\text {suc }}^{\text {avg }}=\left(S^{\prime}+8\right) / 16$. Indeed the classical PAM bound $S^{\prime}=4$ corresponds to $p_{\text {suc }}^{\text {avg }}=$ $3 / 4$, the maximum achievable for this RAC when Alice sends a classical bit with shared randomness. On the other hand, for any quantum violation of $S^{\prime}$, we do have a corresponding advantage in the associated QRAC. Thus, observing any $p_{\text {suc }}^{\text {avg }}>3 / 4$ in a $(2,2) \mapsto 1$ RAC certifies the nonclassicality of the corresponding PAM scenario, or can act as a dimension witness, depending on the available information regarding the devices. Likewise, any violation of $S^{\prime}$ is a quantum advantage in the RAC protocol. But $S^{\prime}$ and $S$ represent the same inequality class, that is, one can be obtained from the other through change of labels. Hence, in light of our nonclassicality activation result, we 
have thus identified sets of four qubit states being prepared by Alice such that any three of them are classical, but altogether offer an advantage in a relevant communication task.

\section{WITNESSING THE CLASSICALITY OF MEASUREMENTS}

As much as quantum states may be seen as resources in diverse correlation scenarios, one may also look the other way around and inquire whether a given set of quantum measurements are useful for unveiling nonclassicality. For the former, we have shown that Theorem 1 allows to certify that a preparation set only generates classical statistics regardless of which and how many PMs are applied. It may, however, be modified to treat measurements as resources, and certify a set of PMs can only given rise to classical statistics for all possible preparations.

Theorem 3 (PMs classicality for all preparations). Let $\mathcal{M}=\left\{\Pi_{b \mid y}\right\}_{b, y}$ be a collection of PMs on $\mathbb{C}^{d}$, and $\mathcal{S}=$ $\left\{\rho_{x}\right\}_{x}$ be a finite collection of pure d-dimensional quantum preparations. For each measurement effect, define operators $O_{b \mid y}$ through

$$
\Pi_{b \mid y}=\eta O_{b \mid y}+(1-\eta) \frac{\mathbf{1}_{d}}{d},
$$

where $\eta$ is the radius of the largest sphere that can be inscribed into the convex hull of the Bloch vectors representing the preparations in $\mathcal{S}$ [cf. Eq. (3)]. If there exists a probability distribution $\pi(\lambda)$ such that all $p(b \mid x, y)=$ $\operatorname{tr}\left(O_{b \mid y} \rho_{x}\right)$ can be written as Eq. (1) for classical communication of dimension $|\mathcal{A}|$, then measurements $\mathcal{M}$ can never manifest nonclassical statistics for sets of quantum preparations.

Proof. Noting that (i) whenever the classicality model (1) may be used to describe the behavior of $\mathcal{S}$ under a given set of measurements, then any preparation set in $\operatorname{conv}(\mathcal{S})$ is also classically reproducible when probed by those same measurements, (ii) that any density operator on the shrunken Bloch sphere of radius $\eta$ can be written as $\rho_{\boldsymbol{u}}^{\eta}=\eta \rho_{\boldsymbol{u}}+(1-\eta) \mathbf{1}_{d} / d$ and is in $\operatorname{conv}(\mathcal{S})$, and (iii) that, for rank-1 projectors, $\operatorname{tr}\left(O_{b \mid y} \rho_{u}^{\eta}\right)=\operatorname{tr}\left(\Pi_{b \mid y} \rho_{\boldsymbol{u}}\right)$, the argument follows analogously to Theorem 1: if by probing operators $O_{b \mid y}$ with a finite set $\mathcal{S}$ of preparations we find out a classical PAM model [Eq. (1)] exists, then by (i) and (ii) the model exists for all $O_{b \mid y}$ and any $\rho_{\boldsymbol{u}}^{\eta}$, which by (iii) means it exists for $\mathcal{M}$ and all pure preparations. Invoking the same reasoning as before, we can extend the result for projections of any rank. Observing that, if $\mathcal{M}$ is classically reproducible for all pure states, it also is for convex combinations of pure states, then this test certifies $\mathcal{M}$ is classically reproducible for all quantum states.
Theorem 3 shows that by probing the behavior of a given set $\mathcal{M}$ of measurements on a finite set of preparations, we may certify $\mathcal{M}$ is PAM classical, meaning it never generates nonclassical statistics in the PAM scenario, regardless what preparation set it acts upon. The choice of the probe preparations $\rho_{x}$ should be done as to maximize $\eta$, similarly to how we have done when choosing the probe measurements in Fig. 2. When those are given, we arrive at the following linear factibility program:

$$
\begin{aligned}
\text { given } & \mathcal{S}, \mathcal{M}, \eta \\
\text { find } & \pi(\lambda) \\
\text { s.t. } & \Pi_{b \mid y}=\eta O_{b \mid y}+(1-\eta) \frac{\mathbf{1}_{d}}{d}, \forall b, y \\
& \operatorname{tr}\left(O_{b \mid y} \rho_{x}\right)=\sum_{a, \lambda} \pi(\lambda) p(a \mid x, \lambda) p(b \mid a, y, \lambda) \\
& \forall b, x, y \\
& \pi(\lambda) \geq 0 \\
& \sum_{\lambda} \pi(\lambda)=1
\end{aligned}
$$

which can also be cast as a maximization program [cf. Eq. (6)] to which the procedure of iteratively exploring the $\lambda$-space may likewise be applied. Withal, analogous considerations to those in Theorem 2 extend this certification of measurement classicality to generalized measurements.

Theorem 4 (POVMs classicality for all preparations). If a generalized measurements set $\mathcal{M}$ is projectivesimulatable for a given amount $t$ of depolarization, and measurements $\phi_{t}(\mathcal{M})$ are certifiably classical for all operators in an inflated, generalized Bloch ball of radius $1 / t$, then $\mathcal{M}$ is classical for all quantum preparations.

Proof. Given a set $\mathcal{S}^{t}=\left\{\rho_{x}^{t}\right\}_{x}=\left[(1 / t)\left\{\rho_{x}-[(1-t) / d]\right.\right.$ $\left.\left.\mathbf{1}_{d}\right\}\right]_{x}$ of probe operators where the $\rho_{x}$ are pure quantum states, Theorem 3 may be employed to determine whether the projective-simulatable $\phi_{t}(\mathcal{M})$ are classical in relation to all operators $\rho^{t}$ on the $1 / t$-radius Bloch sphere where the operators in $\mathcal{S}^{t}$ lie. Observing that $\operatorname{tr}\left[\phi_{t}\left(M_{b \mid y}\right) \rho^{t}\right]=$ $\operatorname{tr}\left[M_{b \mid y} \phi_{t}\left(\rho^{t}\right)\right]=\operatorname{tr}\left(M_{b \mid y} \rho\right), \forall \rho^{t}$, this is equivalent to determining the classicality of generalized measurements $\mathcal{M}$ in relation to any $\rho$, which are all possible quantum preparations.

We finally remark that, although Theorem 3 was stated in regard to pure probes, in principle there is no issue in probing with the $\rho^{t} \in \mathcal{S}^{t}$, as all quantum states are more mixed than these operators. 


\section{A. Incompatible classical measurements}

Quantum formalism is inlaid with the existence of quantities that may not simultaneously be known with arbitrary precision, which is one of many ways it defies our intuition. Any set of measurements with this property is called an incompatible measurement set, with compatibility being the opposite concept. Measurement incompatibility is known to be a necessary but insufficient condition for nonclassicality manifestations in Bell nonlocality [60,61], and it was also shown to be necessary and sufficient for quantum advantage in EPR steering scenarios $[62,63]$. Relations between incompatibility and PAM scenarios with quantum inputs [64] and in the context of ontological models [65] have also been studied. Furthermore, RACs have been proposed as a measure of incompatibility of observables, and it is known that incompatibility is a necessary and sufficient condition for quantum advantage in $2 \mapsto 1$ binary RACs [66,67]. The observations in Sec. III C show us incompatibility is thereby necessary and sufficient for nonclassicality in the aforementioned $|\mathcal{X}|=4$, $|\mathcal{B}|=|\mathcal{A}|=|\mathcal{Y}|=2$ PAM scenario. That sufficiency does not hold in general for PAM scenarios with shared classical randomness will be shown through an application of our measurements classicality certification method.

Measurement compatibility may be understood as joint measurability. Let $\mathcal{M}=\left\{M_{b \mid y}\right\}_{b, y}$ be any set of measurements, with each having the same number of outcomes for convenience. Whenever a $J_{\ell}$, with $\ell=\ell_{1} \ell_{2} \ldots \ell|\mathcal{Y}|$ and each $\ell_{i} \in\{1, \ldots,|\mathcal{B}|\}$, is a positive semidefinite operator such that $\sum_{\ell} J_{\ell}=\mathbf{1}$ and $\sum_{\ell} J_{\ell} \delta_{\ell_{x}, a}=M_{a \mid x}$ then $\mathcal{M}$ is said to be jointly measurable, in the sense that the so-called

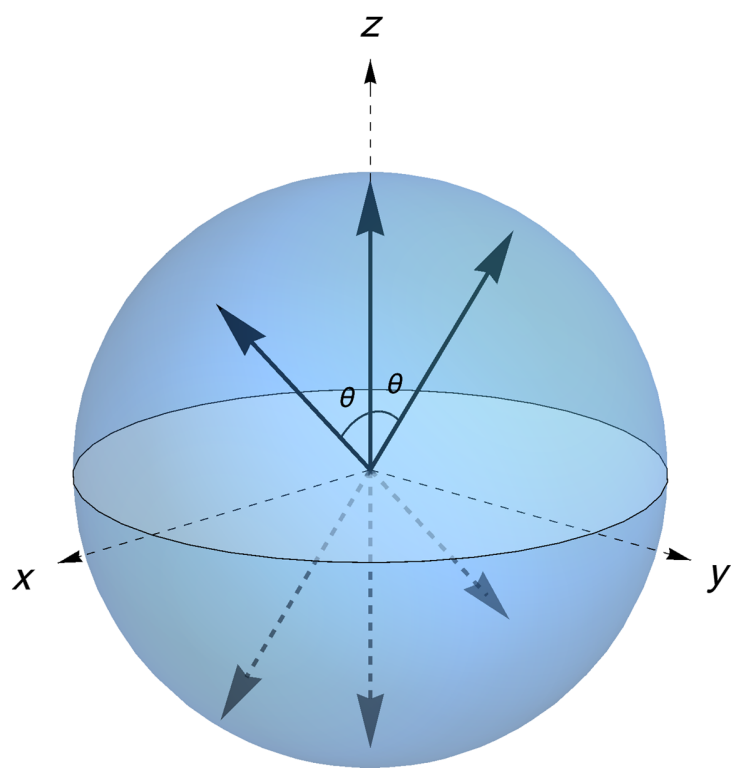

FIG. 6. Mirror-symmetric measurements used to show the existence of incompatible measurements that do not exhibit nonclassical statistics for any preparation set. parent measurement $J_{\ell}$ is a single, well-defined measurement from which every $M_{a \mid x}$ may be recovered. Whenever such a $J_{\ell}$ does not exist, $\mathcal{M}$ is incompatible, or not jointly measurable.

Another invaluable concept is that of incompatibility robustness $\chi_{\mathcal{M}}^{*}$ [68-70], measuring how compatible measurements $\mathcal{M}$ are through

$$
\chi_{\mathcal{M}}^{*}=\sup _{\substack{\chi \in[0,1] \\\left\{N_{b \mid y}\right\} \in \mathbf{N}(\mathcal{M})}}\left\{\chi \mid \chi\left\{M_{b \mid y}\right\}+(1-\chi)\left\{N_{b \mid y}\right\} \in \mathbf{J M}\right\}
$$

In this definition, $\mathbf{J M}$ is the set of jointly measurable measurements, and $\mathbf{N}$ is a noise model that, possibly depending on the $M_{b \mid y}$, determines the noise set, which must contain at least one jointly measurable set of measurements. Given $\mathbf{N}$, the lower the $\chi_{\mathcal{M}}^{*}$, the more incompatible the measurements are, with $\chi_{\mathcal{M}}^{*}=1$ if and only if our measurement set is jointly measurable. For closed noise sets, Eq. (10) turns into a maximization problem that may be written as a semidefinite program (see Appendix E in [70]). This is true, in particular, for the identity noise, $N_{b \mid y}=1 /|\mathcal{B}|$, which is a common choice when unbiased noise is of interest.

Determining $\chi_{\mathcal{M}}^{*}$ thence amounts to choosing a noise model with the required properties and optimizing (10) via semidefinite programming. Defining $\mathcal{M}$ as the mirrorsymmetric measurements shown in Fig. 6 and choosing a random noise map, $\chi_{\mathcal{M}}^{*}$ behaves as shown in Fig. 7. Any value of $\chi$ above the incompatibility robustness curve represents incompatible measurements. Applying program (9)

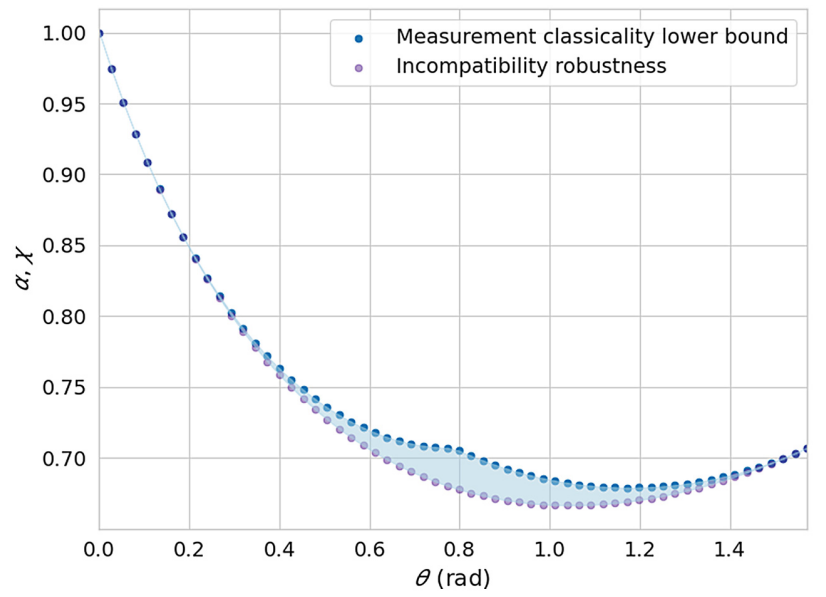

FIG. 7. Incompatibility is insufficient for nonclassicality in the PAM scenario. For each $\theta$ (see Fig. 6), any $\chi$ above the incompatibility robustness curve stands for an incompatible measurement set, and any $\alpha$ below the measurement classicality lower bound represents measurements that certifiedly do not generate nonclassical statistics, regardless what preparations they act upon. Thenceforth, the shaded region contains incompatible albeit classical measurements. 
as a maximization problem to these same measurements, we obtain lower bounds for their classicality, meaning that everything under the measurement classicality curve stands for measurements which are not able to generate nonclassical statistics, irrespective of the preparation set we choose. As, in the shaded region, this is above the incompatibility curve, we conclude there are incompatible measurement sets for which no preparations can exhibit advantage over communicating through two-dimensional classical systems. Put in other words, incompatibility is not, in general, sufficient for nonclassicality in the PAM scenario.

\section{CONCLUSION}

The ability to certify classicality is essential to known applications of the PAM scenario, which range from communication in quantum networks and self-testing of quantum channels to randomness certification and beyond. Although Bell-like inequalities for some modest settings in the PAM scenario were already known, and activation phenomena were found for quantum preparations under two PMs, a method to certify classicality for a scenario with shared randomness and any number of measurements remained elusive.

We have contributed to this problem devising a sufficient criterion to certify classicality for both projective and generalized measurements by only probing the statistics of finitely many measurements, then showing an activation phenomenon indeed happens for all dichotomic measurements in a large set of quantum preparations - a result intimately connected to quantum advantages in RACs. Using an optimization strategy inspired by [50], we were able to increase the number of measurements we probe - an indispensable ingredient to our method - to otherwise intractable values. This was essential to the applications we have shown, and is straightforwardly adaptable to other scenarios such as Bell nonlocality and EPR steering $[28,29]$.

In turn, studying measurements as resources has been an active research topic $[71,72]$, and following this trend we adapted our method to certify a given set of measurements is never able to generate nonclassical behaviors, irrespective of what preparation set they are applied to. We showed the value of this tool by proving there are incompatible measurements which can only lead to classical correlations, which means measurement incompatibility is not sufficient for nonclassicality in the PAM scenario.

Further interesting possibilities are that of activation phenomena under generalized measurements with more than two effects, and activation of measurements nonclassicality (similarly to what we have proven for the nonclassicality activation of states). We were not able to show they happen for our targeted scenarios, but investigating larger settings is an interesting next step to which our methods are readily applicable. On a more conceptual aspect, investigating the relationship between our classicality model and the one studied in the formalism of ontological models could also lead to interesting results. In addition, Figs. 5 and 7 shows our results are both noise and preparation error resistant for a large span of states, and experimental implementation would require no entangled states. Consequently, we believe our results are verifiable in practice.

\section{ACKNOWLEDGMENTS}

C.G. and R.R. would like to thank Carlos Vieira, Marcelo Terra Cunha, and Roberto Baldijão for helpful discussions. The authors also thank R. Uola for useful suggestions. We acknowledge the John Templeton Foundation via the Grant Q-CAUSAL No. 61084, the Serrapilheira Institute (Grant No. Serra-1708-15763), the Brazilian National Council for Scientific and Technological Development $(\mathrm{CNPq})$ via the National Institute for Science and Technology on Quantum Information (INCTIQ), Grants No. 307172/2017-1 and No. 406574/2018-9, the Brazilian agencies MCTIC and MEC, the São Paulo Research Foundation FAPESP (Grant No. 2018/07258-7) and FAEPEX/UNICAMP (Grant No. 3044/19).

[1] J. S. Bell, On the Einstein Podolsky Rosen paradox, Phys. Phys. Fiz. 1, 195 (1964).

[2] N. Brunner, D. Cavalcanti, S. Pironio, V. Scarani, and S. Wehner, Bell nonlocality, Rev. Mod. Phys. 86, 419 (2014).

[3] H. M. Wiseman, S. J. Jones, and A. C. Doherty, Steering, Entanglement, Nonlocality, and the Einstein-PodolskyRosen Paradox, Phys. Rev. Lett. 98, 140402 (2007).

[4] D. Cavalcanti and P. Skrzypczyk, Quantum steering: A review with focus on semidefinite programming, Rep. Prog. Phys. 80, 024001 (2016).

[5] R. Uola, A. C. S. Costa, H. C. Nguyen, and O. Gühne, Quantum steering, Rev. Mod. Phys. 92, 015001 (2020).

[6] R. Gallego, N. Brunner, C. Hadley, and A. Acín, DeviceIndependent Tests of Classical and Quantum Dimensions, Phys. Rev. Lett. 105, 230501 (2010).

[7] J. Bowles, N. Brunner, and M. Pawłowski, Testing dimension and nonclassicality in communication networks, Phys. Rev. A 92, 022351 (2015).

[8] Y. Wang, I. W. Primaatmaja, E. Lavie, A. Varvitsiotis, and C. C. W. Lim, Soundness and completeness of quantum root-mean-square errors, npj Quantum Inf. 5, 1 (2019).

[9] A. Tavakoli, J. Kaniewski, T. Vértesi, D. Rosset, and N. Brunner, Self-testing quantum states and measurements in the prepare-and-measure scenario, Phys. Rev. A 98, 062307 (2018).

[10] N. Miklin and M. Oszmaniec, ArXiv:2003.01032 (2020).

[11] M. Pawłowski and N. Brunner, Semi-device-independent security of one-way quantum key distribution, Phys. Rev. A 84, 010302 (2011). 
[12] E. Passaro, D. Cavalcanti, P. Skrzypczyk, and A. Acín, Optimal randomness certification in the quantum steering and prepare-and-measure scenarios, New J. Phys. 17, 113010 (2015).

[13] H.-W. Li, M. Pawłowski, Z.-Q. Yin, G.-C. Guo, and Z.F. Han, Semi-device-independent randomness certification using $n \rightarrow 1$ quantum random access codes, Phys. Rev. A 85, 052308 (2012).

[14] M. Pawłowski, T. Paterek, D. Kaszlikowski, V. Scarani, A. Winter, and M. Żukowski, Information causality as a physical principle, Nature 461, 1101 (2009).

[15] R. Chaves, C. Majenz, and D. Gross, Information-theoretic implications of quantum causal structures, Nat. Commun. 6, 1 (2015).

[16] R. Chaves, G. B. Lemos, and J. Pienaar, Causal Modeling the Delayed-Choice Experiment, Phys. Rev. Lett. 120, 190401 (2018).

[17] A. S. Holevo, Bounds for the quantity of information transmitted by a quantum communication channel, Probl. Peredachi Inf. 9, 3 (1973).

[18] J. Bowles, M. T. Quintino, and N. Brunner, Certifying the Dimension of Classical and Quantum Systems in a Prepare-And-Measure Scenario with Independent Devices, Phys. Rev. Lett. 112, 140407 (2014).

[19] R. Chaves, J. B. Brask, and N. Brunner, DeviceIndependent Tests of Entropy, Phys. Rev. Lett. 115, 110501 (2015).

[20] T. Van Himbeeck, E. Woodhead, N. J. Cerf, R. GarcíaPatrón, and S. Pironio, Semi-device-independent framework based on natural physical assumptions, Quantum 1, 33 (2017).

[21] A. Tavakoli, E. Z. Cruzeiro, J. B. Brask, N. Gisin, and N. Brunner, Informationally restricted quantum correlations, Quantum 4, 332 (2020).

[22] D. Poderini, S. Brito, R. Nery, F. Sciarrino, and R. Chaves, Criteria for nonclassicality in the prepare-and-measure scenario, Phys. Rev. Res. 2, 043106 (2020).

[23] N. Brunner, M. Navascués, and T. Vértesi, Dimension Witnesses and Quantum State Discrimination, Phys. Rev. Lett. 110, 150501 (2013).

[24] M. Dall'Arno, E. Passaro, R. Gallego, and A. Acin, Robustness of device-independent dimension witnesses, Phys. Rev. A 86, 042312 (2012).

[25] G. Moreno, R. Nery, C. de Gois, R. Rabelo, and R. Chaves, Semi-device-independent certification of entanglement in superdense coding, Phys. Rev. A 103, 022426 (2021).

[26] R. F. Werner, Quantum states with Einstein-PodolskyRosen correlations admitting a hidden-variable model, Phys. Rev. A 40, 4277 (1989).

[27] J. Barrett, Nonsequential positive-operator-valued measurements on entangled mixed states do not always violate a bell inequality, Phys. Rev. A 65, 042302 (2002).

[28] D. Cavalcanti, L. Guerini, R. Rabelo, and P. Skrzypczyk, General Method for Constructing Local Hidden Variable Models for Entangled Quantum States, Phys. Rev. Lett. 117, 00 (2016).

[29] F. Hirsch, M. T. Quintino, T. Vértesi, M. F. Pusey, and N. Brunner, Algorithmic Construction of Local Hidden Variable Models for Entangled Quantum States, Phys. Rev. Lett. 117, 190402 (2016).
[30] S. Popescu, Bell's Inequalities and Density Matrices: Revealing "Hidden" Nonlocality, Phys. Rev. Lett. 74, 2619 (1995).

[31] F. Hirsch, M. T. Quintino, J. Bowles, and N. Brunner, Genuine Hidden Quantum Nonlocality, Phys. Rev. Lett. 111, 160402 (2013).

[32] R. Gallego, L. E. Würflinger, R. Chaves, A. Acín, and M. Navascués, Nonlocality in sequential correlation scenarios, New J. Phys. 16, 033037 (2014).

[33] M. Navascués and T. Vértesi, Activation of Nonlocal Quantum Resources, Phys. Rev. Lett. 106, 060403 (2011).

[34] C. Palazuelos, Superactivation of Quantum Nonlocality, Phys. Rev. Lett. 109, 190401 (2012).

[35] D. Cavalcanti, M. L. Almeida, V. Scarani, and A. Acin, Quantum networks reveal quantum nonlocality, Nat. Commun. 2, 1 (2011).

[36] R. W. Spekkens, D. H. Buzacott, A. J. Keehn, B. Toner, and G. J. Pryde, Preparation Contextuality Powers ParityOblivious Multiplexing, Phys. Rev. Lett. 102, 010401 (2009).

[37] R. Kunjwal and R. W. Spekkens, From the KochenSpecker Theorem to Noncontextuality Inequalities Without Assuming Determinism, Phys. Rev. Lett. 115, 110403 (2015).

[38] M. D. Mazurek, M. F. Pusey, R. Kunjwal, K. J. Resch, and R. W. Spekkens, An experimental test of noncontextuality without unphysical idealizations, Nat. Commun. 7, 1 (2016).

[39] D. Schmid, R. W. Spekkens, and E. Wolfe, All the noncontextuality inequalities for arbitrary prepare-and-measure experiments with respect to any fixed set of operational equivalences, Phys. Rev. A 97, 062103 (2018).

[40] F. Shahandeh, Contextuality of general probabilistic theories, PRX Quantum 2, 010330 (2021).

[41] D. Schmid, J. H. Selby, E. Wolfe, R. Kunjwal, and R. W. Spekkens, Characterization of noncontextuality in the framework of generalized probabilistic theories, PRX Quantum 2, 010331 (2021).

[42] V. Gitton and M. P. Woods, ArXiv:2003.06426 (2020).

[43] D. Schmid and R. W. Spekkens, Contextual Advantage for State Discrimination, Phys. Rev. X 8, 011015 (2018).

[44] M. J. W. Hall, Local Deterministic Model of Singlet State Correlations Based on Relaxing Measurement Independence, Phys. Rev. Lett. 105, 250404 (2010).

[45] C. J. Wood and R. W. Spekkens, The lesson of causal discovery algorithms for quantum correlations: Causal explanations of bell-inequality violations require fine-tuning, New J. Phys. 17, 033002 (2015).

[46] R. Chaves, R. Kueng, J. B. Brask, and D. Gross, Unifying Framework for Relaxations of the Causal Assumptions in Bell's Theorem, Phys. Rev. Lett. 114, 140403 (2015).

[47] A. Fine, Hidden Variables, Joint Probability, and the Bell Inequalities, Phys. Rev. Lett. 48, 291 (1982).

[48] M. Oszmaniec, L. Guerini, P. Wittek, and A. Acín, Simulating Positive-Operator-Valued Measures with Projective Measurements, Phys. Rev. Lett. 119, 00 (2017).

[49] F. Hirsch, M. T. Quintino, T. Vértesi, M. Navascués, and N. Brunner, Better local hidden variable models for two-qubit 
Werner states and an upper bound on the Grothendieck constant KG(3), Quantum 1, 3 (2017).

[50] M. Fillettaz, F. Hirsch, S. Designolle, and N. Brunner, Algorithmic construction of local models for entangled quantum states: Optimization for two-qubit states, Phys. Rev. A 98, 00 (2018).

[51] R. T. Rockafellar, Convex Analysis (Princeton University Press, Princeton, 1970), Vol. 28.

[52] See https://github.com/cgois/pam classicality.

[53] S. Wiesner, ACM Sigact News 15, 78 (1983).

[54] A. Ambainis, A. Nayak, A. Ta-Shma, and U. Vazirani, in Proceedings of the Thirty-First Annual ACM Symposium on Theory of Computing (Association for Computing Machinery, New York, 1999), p. 376.

[55] A. Nayak, in 40th Annual Symposium on Foundations of Computer Science (Cat. No. 99CB37039) (IEEE, New York, 1999), p. 369.

[56] A. Ambainis, A. Nayak, A. Ta-Shma, and U. Vazirani, Dense quantum coding and quantum finite automata, J. ACM (JACM) 49, 496 (2002).

[57] M. Hayashi, K. Iwama, H. Nishimura, R. Raymond, and S. Yamashita, (4,1)-Quantum random access coding does not exist - one qubit is not enough to recover one of four bits, New J. Phys. 8, 129 (2006).

[58] A. Ambainis, D. Leung, L. Mancinska, and M. Ozols, ArXiv:0810.2937 (2008).

[59] M. Czechlewski, D. Saha, A. Tavakoli, and M. Pawłowski, Device-independent witness of arbitrary-dimensional quantum systems employing binary-outcome measurements, Phys. Rev. A 98, 062305 (2018).

[60] E. Bene, and T. Vértesi, Measurement incompatibility does not give rise to Bell violation in general, New J. Phys. 20, 013021 (2018).
[61] F. Hirsch, M. T. Quintino, and N. Brunner, Quantum measurement incompatibility does not imply Bell nonlocality, Phys. Rev. A 97, 012129 (2018).

[62] R. Uola, T. Moroder, and O. Gühne, Joint Measurability of Generalized Measurements Implies Classicality, Phys. Rev. Lett. 113, 160403 (2014).

[63] M. T. Quintino, T. Vértesi, and N. Brunner, Phys. Rev. Lett. 113, 160402 (2014).

[64] L. Guerini, M. T. Quintino, and L. Aolita, Distributed sampling, quantum communication witnesses, and measurement incompatibility, Phys. Rev. A 100, 042308 (2019).

[65] A. Tavakoli, and R. Uola, Measurement incompatibility and steering are necessary and sufficient for operational contextuality, Phys. Rev. Res. 2, 013011 (2020).

[66] D. Saha, M. Oszmaniec, L. Czekaj, M. Horodecki, and R. Horodecki, Operational foundations for complementarity and uncertainty relations, Phys. Rev. A 101, 052104 (2020).

[67] C. Carmeli, T. Heinosaari, and A. Toigo, EPL (Europhys. Lett.) 130, 50001 (2020).

[68] R. Uola, C. Budroni, O. Gühne, and J.-P. Pellonpää, One-to-One Mapping between Steering and Joint Measurability Problems, Phys. Rev. Lett. 115, 230402 (2015).

[69] E. Haapasalo, Robustness of incompatibility for quantum devices, J. Phys. A: Math. Theor. 48, 255303 (2015).

[70] S. Designolle, M. Farkas, and J. Kaniewski, Incompatibility robustness of quantum measurements: a unified framework, New J. Phys. 21, 113053 (2019).

[71] M. Oszmaniec, and T. Biswas, Quantum 3, 133 (2019).

[72] F. Buscemi, E. Chitambar, and W. Zhou, Phys. Rev. Lett. 124, 120401 (2020). 